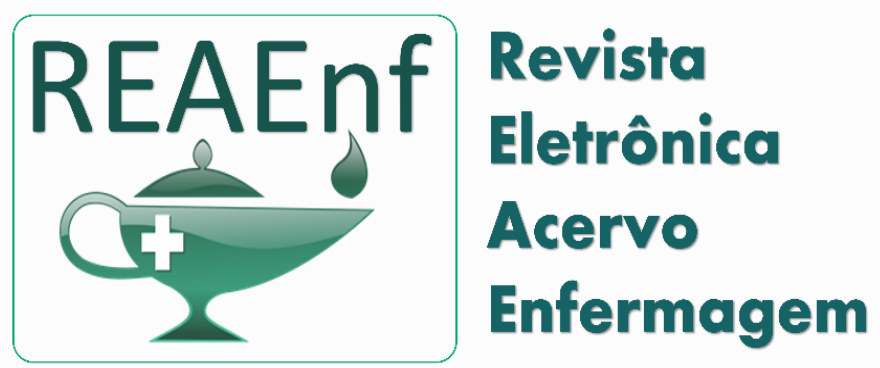

REVISÃO BIBLIOGRÁFICA

Recebido em: 12/2020

Aceito em: $1 / 2021$

Publicado em: $3 / 2021$

\title{
Repercussões biopsicossociais na vida de jovens e adultos colostomizados
}

\author{
Biopsychosocial repercussions on the lives of colostomized youth and adults
}

Repercusiones biopsicosociales en la vida de jóvenes y adultos colostomizados

Emillie Gagliardi Bitencourt" ${ }^{1 \star}$ Neyviton Silva ${ }^{1}$, Barbara Jacqueline Peres Barbosa ${ }^{1}$.

\begin{abstract}
Resumo: Este estudo é uma revisão integrativa com abordagem descritiva que tem como objetivo apresentar a qualidade de vida e os desafios enfrentados por jovens e adultos ao decorrer de suas vidas, após serem submetidos à confecção de uma estomia intestinal, visto que existe uma série de adversidades envolvidas no processo de desenvolvimento e amadurecimento dos seres humanos. Em especial quando esse processo envolve mudanças drásticas, como a confecção de um estoma, esses, correlacionam-se com os aspectos biológicos, psicológicos e sociais, relacionado às consequências de alterações anatômicas, repercussões na autoimagem e autoestima e no contexto social, trazendo dificuldades de reinserção no mercado de trabalho. No tocante à enfermagem, a equipe necessita de educação permanente no que diz respeito aos cuidados com a colostomia e abordagem humanizada; permitindo que o paciente se sinta acolhido e encorajado a esclarecer suas dúvidas e expressar seus sentimentos, possibilitando maior disposição para o enfrentamento de um novo normal.
\end{abstract}

Palavra-chave: Colostomia, Enfermagem, Qualidade de vida.

Abstract: This study is an integrative review with a descriptive approach that aims to present the quality of life and the challenges faced by young people and adults throughout their lives, after being submitted to an intestinal ostomy, since there is a series of adversities involved in the process of development and maturation of human beings. Especially when this process involves drastic changes, such as the construction of a stoma, these correlate with biological, psychological and social aspects, related to the consequences of anatomical changes, repercussions on self-image and self-esteem and in the social context, bringing difficulties of reintegration into the labor market. With regard to nursing, the team needs permanent education with regard to colostomy care and a humanized approach; allowing the patient to feel welcomed and encouraged to clarify his doubts and express his feelings, enabling greater willingness to face a new normal.

Key word: Colostomy, Nursing, Quality of life.

Resumen: Este estudio es una revisión integradora con un enfoque descriptivo que tiene como objetivo presentar la calidad de vida y los desafíos que enfrentan los jóvenes y adultos a lo largo de su vida, luego de ser sometidos a la construcción de una ostomía intestinal, ya que hay una serie de adversidades que intervienen en el proceso de desarrollo y maduración del ser humano. Especialmente cuando este proceso implica cambios drásticos, como la construcción de un estoma, estos se correlacionan con aspectos biológicos, psicológicos y sociales, relacionados con las consecuencias de los cambios anatómicos, repercusiones en la autoimagen y autoestima y en el contexto social, trayendo dificultades de reintegración al mercado laboral. En enfermería, el equipo necesita educación permanente en el cuidado de la colostomía y un enfoque humanizado; permitiendo que el paciente se sienta acogido y animado para aclarar sus dudas y expresar sus sentimientos, posibilitando una mayor disposición para afrontar una nueva normalidad.

Palabras clave: Colostomía, Enfermería, Calidad de vida.

${ }^{1}$ Universidade Anhembi Morumbi (UAM), São Paulo - SP. *E-mail: emillie.g.b@hotmail.com

REAEnf/EJNC | Vol. 10 | e6166 | DOI: https://doi.org/10.25248/REAenf.e6166.2021 Página 1 de 11 


\section{INTRODUÇÃO}

A colostomia e os demais estomas digestivos são realizados com fins terapêuticos, visando associar sobrevida e qualidade de vida aos indivíduos estomizados em diferentes doenças, como as Doenças Inflamatórias Intestinais (DII), entre as quais estão a doença de Crohn e a colite ulcerosa, doenças hereditárias como a polipose familiar, as doenças congênitas (megacólon congênito) e os traumas abdominais perfurantes, sendo a principal causa para realização do estômato o câncer colorretal que correspondeu por certa de 41 mil diagnósticos no ano de 2018 (BONILL-DE-LAS-NIEVES C, et al., 2014; MINISTÉRIO DA SAÚDE (MS), 2020).

A Organização Mundial da Saúde (OMS) afirma que os indivíduos Ostomizados podem chegar a 0,1\% da população do planeta. Aparentemente parece uma quantidade ínfima, porém, no Brasil 100 mil pessoas já realizaram uma ostomia. Essa cirurgia tem como objetivo proporcionar sobrevida e qualidade de vida aos indivíduos estomizados (ESTIMA R, 2004; VIEIRA LM, et al., 2013).

O termo estomia tem origem na palavra grega stóma, boca, construída por meio de intervenção cirúrgica, com o objetivo de exteriorização das vísceras ocas. A história das estomias é conhecida desde o ano de 350 a.C. onde Praxógoras de Kos teria realizado esse procedimento em um caso de ferimento abdominal. Em 1776, Pillore, um cirurgião de Rouen, na França, realizou com sucesso uma cirurgia que consistiu na sutura do ceco intestinal na parede abdominal, este procedimento é conhecido como cecostomia inguinal. Em 1783, um cirurgião de Napoleão, Antoine Dubois, relata a realização de uma colostomia em uma criança de três dias de vida com imperfuração anal (BACELAR S, et al., 2014; CASCAIS AFMV, et al., 2007).

Entre as estomias, a confecção da colostomia, que é um procedimento cirúrgico de abertura do segmento cólico na parede abdominal, tem maior destaque e pode ser realizada de forma eletiva ou emergencial, podendo ainda ter como critério de classificação a possibilidade de reversão, ou seja, colostomia de caráter reversível ou irreversível (MEIREILES CA, et al., 2001).

Um desafio ainda maior é conviver com um estoma, sendo jovem e inserido num contexto social onde falta educação e orientação à população a respeito das necessidades de uma pessoa estomizada, bem como políticas e adaptações nos ambientes que propiciem a socialização desses pacientes (SELAU CM, et al., 2019).

Existem as especificidades quando a colostomia é confeccionada em indivíduos jovens, já que essa fase é um processo deveras complexo e que implica em mudanças corporais, psicológicas e comportamentais. Afinal, ser jovem por si só é um completo desafio, além da necessidade de se adequar a um grupo e ser aceito como indivíduo, pode trazer sofrimento psíquico, fazendo com que o indivíduo tente mudar ou esconder características que os definem, levando ao isolamento social, visto que conviver com uma colostomia pode trazer alguns constrangimentos com relação a suas eliminações e higiene (RAITZ TR e PETTERS LCF, 2008).

Os desafios de se adaptar a uma nova estrutura corporal, com um processo diferente do fisiológico para realização das eliminações intestinais, tornam as atividades de vida diária (AVDs) um tanto complexas em detrimento aos demais indivíduos, além disso, um novo orifício pode trazer curiosidades que potencializam os riscos de sérias complicações, como exemplo, a penetração do estoma no ato sexual (FREIRE DA, et al., 2017).

A nova realidade enfrentada pelos estomizados se mostra conflituosa em todos os aspectos, principalmente no psicossocial. Comumente o indivíduo se sente muito diferente dos demais até mesmo excluído. O indivíduo colostomizado sofre um abalo psicológico, físico, assim como, uma destruição súbita de sua imagem corporal (VIEIRA LM, et al., 2013; BATISTA MRFF, et al., 2011).

A partir disso torna-se indispensável o papel do enfermeiro como agente promotor da prevenção, educar e sanar dúvidas, para garantir que o paciente tenha consciência e orientação a respeito dos seus atos e consequências, assim o indivíduo ingressará para um novo normal com mais segurança e sentindo-se amparado, o que implicará em uma maior qualidade de vida (NASCIMENTO CMS, et al., 2011).

O presente artigo busca mostrar por meio desta revisão os desafios enfrentados por jovens e adultos no decorrer de suas vidas, após serem submetidos à confecção da colostomia. 


\section{MÉTODOS}

Trata-se de uma revisão integrativa da literatura científica, com abordagem descritiva, onde foi possível sintetizar as pesquisas disponíveis sobre as repercussões biopsicossociais na vida de jovens e adultos colostomizados, possibilitando a compreensão das mudanças enfrentadas por esses indivíduos (SOUZA MT, 2010; DURAN ECM, 2011). A pesquisa foi realizada por meio do levantamento de artigos científicos qualitativos e quantitativos e a coleta de dados ocorreu entre os meses de setembro a novembro de 2020.

As bases de dados pesquisadas foram Biblioteca Virtual em Saúde - BVS e demais cooperadores: Literatura Latino-Americana e do Caribe em Ciências da Saúde (LILACS), Scientific Electronic Library Online (SciELO) e Banco de Dados em Enfermagem (BDENF). Utilizaram-se, como descritores controlados, identificados nos Descritores em Ciência da Saúde (DECs): "colostomia", "qualidade de vida", "enfermagem", "Colostomy", "qualityoflife", "nursing".

A estratégia de busca mediante o operador booleano AND e OR foi: "colostomia and qualidade de vida and enfermagem", "colostomia and enfermagem", "qualidade de vida and enfermagem", e ocorreu no idioma português. Foram incluídos artigos de pesquisa originais, cuja temática respondesse à pergunta norteadora, publicados nos últimos 20 anos, na língua portuguesa, disponíveis on-line e na íntegra. Excluíram-se estudos que não tinham metodologia de pesquisa (relatos de caso, reflexões, recomendações), as revisões, os estudos que focavam outras temáticas. Os estudos foram também incluídos, por considerar a limitação no número de estudos com a população estudada, no intuito de alcançar o máximo de informações sobre essa população. Conforme representado na Figura 1.

Figura 1 - Fluxograma PRISMA das etapas de pesquisa para realização dessa revisão integrativa.

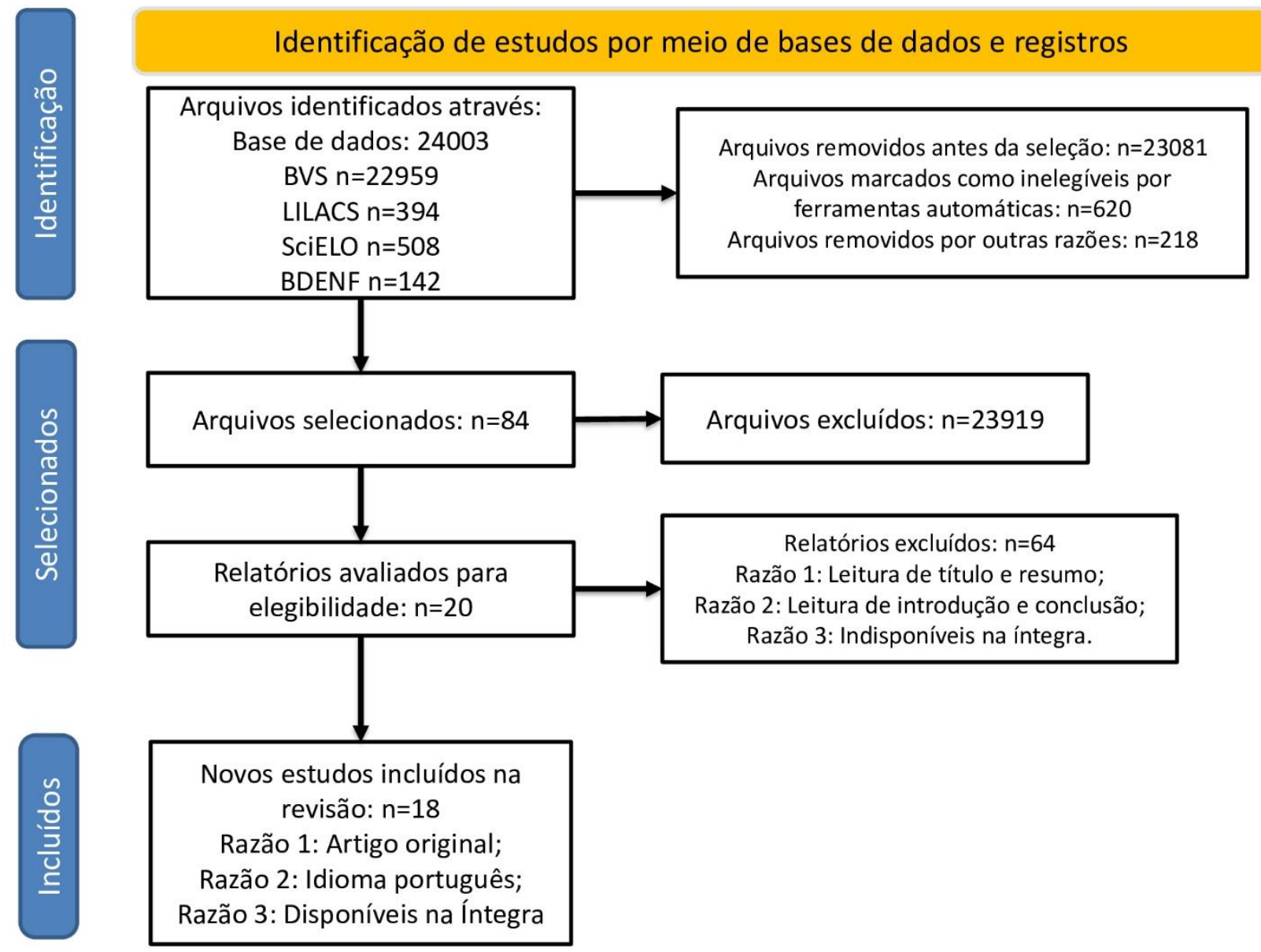

Fonte: Bitencourt EG, et al., 2020 


\section{RESULTADOS E DISCUSSÃO}

Os estudos analisados foram realizados no período de 2000 a 2019. Destes,78\% foram publicados no Brasil, um era proveniente da Espanha $(n=4)$ e um era proveniente de Portugal $(n=11)$. Quanto à área de inserção dos autores, todos eram provenientes das áreas de Enfermagem, Medicina, Psicologia ou Fisioterapia, conforme mostra a Tabela 1. 
Tabela 1 - Caracterização dos artigos incluídos na pesquisa.

\begin{tabular}{|c|c|c|c|c|c|c|c|}
\hline $\mathbf{N}$ & Título & Autores & Ano & Periódico & Objetivo Geral & $\begin{array}{c}\text { Tipo de } \\
\text { Pesquisa }\end{array}$ & País \\
\hline 1 & $\begin{array}{l}\text { Ostomia, uma difícil } \\
\text { adaptação }\end{array}$ & $\begin{array}{l}\text { Barbutti RCS, et } \\
\text { al. }\end{array}$ & 2008 & Rev. SBPH & $\begin{array}{l}\text { Identificar alterações causadas pela } \\
\text { confecção de uma ostomia, em uma } \\
\text { visão geral sobre o processo de viver } \\
\text { do paciente ostomizado. }\end{array}$ & $\begin{array}{c}\text { Estudo } \\
\text { Observacional }\end{array}$ & Brasil \\
\hline 2 & $\begin{array}{l}\text { Aspectos clínicos e } \\
\text { epidemiológicos de } \\
\text { estomizados intestinais de } \\
\text { um município de Minas } \\
\text { Gerais }\end{array}$ & $\begin{array}{c}\text { Barbosa } \mathrm{MH} \text {, et } \\
\text { al. }\end{array}$ & 2014 & $\begin{array}{l}\text { Revista de } \\
\text { Enfermagem e } \\
\text { Atenção à } \\
\text { Saúde }\end{array}$ & $\begin{array}{l}\text { Caracterizar pacientes estomizados } \\
\text { intestinais atendidos no programa de } \\
\text { atenção multiprofissional ao paciente } \\
\text { estomizado do município de Uberaba } \\
\text { MG quanto aos aspectos } \\
\text { sociodemográficos e clínicos }\end{array}$ & $\begin{array}{l}\text { Descritivo } \\
\text { Exploratório } \\
\text { Quantitativo }\end{array}$ & Brasil \\
\hline 3 & $\begin{array}{l}\text { Autoimagem de clientes } \\
\text { com colostomia em } \\
\text { relação a bolsa coletora }\end{array}$ & $\begin{array}{c}\text { Batista MRFF, } \\
\text { et al. }\end{array}$ & 2011 & $\begin{array}{l}\text { Rev. Bras } \\
\text { Enferm }\end{array}$ & $\begin{array}{l}\text { Analisar a percepção do portado de } \\
\text { colostomia em relação ao uso da } \\
\text { bolsa coletora }\end{array}$ & $\begin{array}{l}\text { Descritivo } \\
\text { Qualitativo }\end{array}$ & Brasil \\
\hline 4 & $\begin{array}{l}\text { Convivendo com estomas } \\
\text { digestivos: estratégias de } \\
\text { enfrentamento da nova } \\
\text { realidade física }\end{array}$ & $\begin{array}{l}\text { Bonill-de-las- } \\
\text { Nieves C, et al. }\end{array}$ & 2014 & $\begin{array}{l}\text { Rev. Latino-Am } \\
\text { Enfermagem }\end{array}$ & $\begin{array}{c}\text { Descrever as estratégias } \\
\text { desenvolvidas pelas pessoas } \\
\text { portadoras de estomas digestivos } \\
\text { para enfrentar a sua situação. }\end{array}$ & $\begin{array}{l}\text { Descritivo } \\
\text { Qualitativo }\end{array}$ & Espanha \\
\hline 5 & $\begin{array}{l}\text { O impacto da ostomia no } \\
\text { processo de viver humano }\end{array}$ & $\begin{array}{c}\text { Cascais AFMV, } \\
\text { et al. }\end{array}$ & 2007 & $\begin{array}{c}\text { Texto Contexto } \\
\text { Enferm }\end{array}$ & $\begin{array}{c}\text { Dar uma visão geral dos estudos } \\
\text { existentes acerca do processo de } \\
\text { viver da pessoa ostomizada }\end{array}$ & $\begin{array}{c}\text { Estudo } \\
\text { Observacional }\end{array}$ & Brasil \\
\hline 6 & $\begin{array}{l}\text { A estomia mudando a } \\
\text { vida: enfrentar para viver }\end{array}$ & $\begin{array}{l}\text { Coelho AR, et } \\
\text { al. }\end{array}$ & 2013 & $\begin{array}{l}\text { Rev. Min } \\
\text { Enferm }\end{array}$ & $\begin{array}{c}\text { Descrever as mudanças ocorridas no } \\
\text { cotidiano do estomizado e identificar } \\
\text { quais as formas de enfrentamento } \\
\text { utilizadas por ele. }\end{array}$ & $\begin{array}{l}\text { Descritivo } \\
\text { Exploratório } \\
\text { Qualitativo }\end{array}$ & Brasil \\
\hline 7 & $\begin{array}{c}\text { O significado da } \\
\text { sexualidade para pessoa } \\
\text { com estoma intestinal } \\
\text { definitivo }\end{array}$ & $\begin{array}{c}\text { De Paula MAB, } \\
\text { et al. }\end{array}$ & 2009 & $\begin{array}{l}\text { Rev. Bras } \\
\text { Coloproct }\end{array}$ & $\begin{array}{c}\text { Identificar a Representação Social da } \\
\text { pessoa com estoma intestinal sobre } \\
\text { a sexualidade. }\end{array}$ & $\begin{array}{l}\text { Descritivo } \\
\text { Exploratório } \\
\text { Qualitativo }\end{array}$ & Brasil \\
\hline
\end{tabular}

REAEnf/EJNC | Vol. 10 | e6166 | DOI: https://doi.org/10.25248/REAenf.e6166.2021 Página $\mathbf{5}$ de 11 


\begin{tabular}{|c|c|c|c|c|c|c|c|}
\hline $\mathbf{N}$ & Título & Autores & Ano & Periódico & Objetivo Geral & $\begin{array}{l}\text { Tipo de } \\
\text { Pesquisa }\end{array}$ & País \\
\hline 8 & $\begin{array}{c}\text { Subsídios para } \\
\text { compreensão da } \\
\text { sexualidade do parceiro } \\
\text { do sujeito portador de } \\
\text { colostomia definitiva }\end{array}$ & $\begin{array}{l}\text { Freitas MRI e } \\
\text { Pelá NTR }\end{array}$ & 2000 & $\begin{array}{l}\text { Rev. Latino-Am } \\
\text { Enfermagem }\end{array}$ & $\begin{array}{l}\text { Descrever como interagem } \\
\text { sexualmente o parceiro sexual e o } \\
\text { sujeito portador de colostomia } \\
\text { definitiva }\end{array}$ & $\begin{array}{l}\text { Descritivo } \\
\text { Qualitativo }\end{array}$ & Brasil \\
\hline 9 & $\begin{array}{c}\text { A sexualidade do paciente } \\
\text { estomizado no discurso do } \\
\text { enfermeiro }\end{array}$ & $\begin{array}{c}\text { Goulart MB, et } \\
\text { al. }\end{array}$ & 2017 & $\begin{array}{l}\text { Rev. Min } \\
\text { Enferm }\end{array}$ & $\begin{array}{l}\text { Identificar a abordagem da } \\
\text { sexualidade no período } \\
\text { perioperatório do paciente } \\
\text { ostomizado, entre os profissionais de } \\
\text { um hospital público de ensino }\end{array}$ & $\begin{array}{l}\text { Descritivo } \\
\text { Qualitativo }\end{array}$ & Brasil \\
\hline 10 & $\begin{array}{l}\text { O enfermeiro e sua } \\
\text { participação no processo } \\
\text { de reabilitação da pessoa } \\
\text { com estoma }\end{array}$ & $\begin{array}{c}\text { Maurício VC, et } \\
\text { al. }\end{array}$ & 2013 & Esc Anna Nery & $\begin{array}{l}\text { Discutir a partir do ponto de vista do } \\
\text { estomizados as orientações } \\
\text { fornecidas pelos enfermeiros em } \\
\text { relação a inclusão laboral }\end{array}$ & $\begin{array}{l}\text { Descritiva } \\
\text { Exploratória } \\
\text { Qualitativa }\end{array}$ & Brasil \\
\hline 11 & $\begin{array}{l}\text { Qualidade de vida da } \\
\text { pessoa estomizada: } \\
\text { relação com os cuidados } \\
\text { prestados na consulta de } \\
\text { enfermagem com a } \\
\text { estomaterapia }\end{array}$ & $\begin{array}{c}\text { Miranda LSG, et } \\
\text { al. }\end{array}$ & 2018 & Esc Anna Nery & $\begin{array}{c}\text { Analisar a relação entre a Qualidade } \\
\text { de Vida (QV) e os cuidados } \\
\text { prestados na consulta de } \\
\text { enfermagem de estomaterapia. }\end{array}$ & $\begin{array}{l}\text { Descritivo } \\
\text { Correlacional }\end{array}$ & Portugal \\
\hline 12 & $\begin{array}{l}\text { Cuidado e saúde em } \\
\text { pacientes estomizados }\end{array}$ & Oliveira IV, et al. & 2018 & $\begin{array}{c}\text { Rev. Bras } \\
\text { Promoç Saúde }\end{array}$ & $\begin{array}{c}\text { Descrever os aspectos } \\
\text { epidemiológicos, as complicações e } \\
\text { as hospitalizações relacionadas à } \\
\text { estomia }\end{array}$ & $\begin{array}{l}\text { Transversal } \\
\text { Descritivo } \\
\text { Quantitativo }\end{array}$ & Brasil \\
\hline 13 & $\begin{array}{c}\text { Assistência de } \\
\text { enfermagem a paciente } \\
\text { com colostomia: aplicação } \\
\text { da teoria de Orem* }\end{array}$ & $\begin{array}{l}\text { Sampaio FAA, } \\
\text { et al. }\end{array}$ & 2008 & $\begin{array}{l}\text { ACTA Paul } \\
\text { Enferm }\end{array}$ & $\begin{array}{c}\text { Aplicar a teoria do autocuidado de } \\
\text { orem na assistência a Paciente } \\
\text { portadora de estomia }\end{array}$ & $\begin{array}{l}\text { Estudo de } \\
\text { Caso }\end{array}$ & Brasil \\
\hline
\end{tabular}

REAEnf/EJNC | Vol. 10 | e6166 | DOI: https://doi.org/10.25248/REAenf.e6166.2021 Página 6 de 11 


\begin{tabular}{|c|c|c|c|c|c|c|c|}
\hline $\mathbf{N}$ & Título & Autores & Ano & Periódico & Objetivo Geral & $\begin{array}{l}\text { Tipo de } \\
\text { Pesquisa }\end{array}$ & País \\
\hline 14 & $\begin{array}{l}\text { Percepção dos cônjuges } \\
\text { de pessoas com estomia } \\
\text { intestinal sobre a } \\
\text { sexualidade do casal }\end{array}$ & Santos FS, et al. & 2019 & $\begin{array}{l}\text { Rev. Min } \\
\text { Enferm }\end{array}$ & $\begin{array}{l}\text { Identificar a percepção de cônjuges } \\
\text { de pessoas com estomia intestinal } \\
\text { sobre a sexualidade do casal }\end{array}$ & $\begin{array}{l}\text { Descritvo } \\
\text { Qualitativo }\end{array}$ & Brasil \\
\hline 15 & $\begin{array}{l}\text { O significado da mudança } \\
\text { no modo de vida da } \\
\text { pessoa com estomia } \\
\text { intestinal definitiva }\end{array}$ & $\begin{array}{l}\text { Silva AL e } \\
\text { Shimizu HE }\end{array}$ & 2006 & $\begin{array}{l}\text { Rev. Latino-Am } \\
\text { Enfermagem }\end{array}$ & $\begin{array}{l}\text { Identificar e analisar as principais } \\
\text { modificações que ocorrem no modo } \\
\text { de vida do portador de estomia } \\
\text { intestinal definitiva para enfrentar a } \\
\text { situação de ser estomizado. }\end{array}$ & $\begin{array}{l}\text { Estudo de } \\
\text { Caso }\end{array}$ & Brasil \\
\hline 16 & $\begin{array}{l}\text { Protocolo de enfermagem } \\
\text { para as alterações } \\
\text { psicossociais e espirituais } \\
\text { da pessoa com colostomia }\end{array}$ & Silva ES, et al. & 2015 & $\begin{array}{l}\text { Cogitare } \\
\text { Enferm }\end{array}$ & $\begin{array}{l}\text { Elaborar um protocolo de assistência } \\
\text { de enfermagem contendo os } \\
\text { diagnósticos/resultados e } \\
\text { intervenções de enfermagem, } \\
\text { relacionados às necessidades } \\
\text { psicossociais e psicoespirituais da } \\
\text { pessoa com colostomia }\end{array}$ & $\begin{array}{l}\text { Descritivo } \\
\text { Exploratório }\end{array}$ & Brasil \\
\hline 7 & $\begin{array}{l}\text { A visão do colostomizado } \\
\text { sobre o uso da bolsa de } \\
\text { colostomia }\end{array}$ & $\begin{array}{l}\text { Sonobe HM, et } \\
\text { al. }\end{array}$ & 2000 & $\begin{array}{l}\text { Revista } \\
\text { Brasileira de } \\
\text { Cancerologia }\end{array}$ & $\begin{array}{l}\text { Identificar a visão do colostomizado } \\
\text { sobre o uso da bolsa de colostomia }\end{array}$ & $\begin{array}{l}\text { Exploratório } \\
\text { Qualitativo }\end{array}$ & Brasil \\
\hline 18 & $\begin{array}{l}\text { Câncer colorretal dois } \\
\text { pontos entro o sofrimento } \\
\text { e o repensar na vida }\end{array}$ & Vieira LM, et al. & 2013 & $\begin{array}{l}\text { Saúde em } \\
\text { Debate }\end{array}$ & $\begin{array}{l}\text { Compreender as experiências vividas } \\
\text { por } 6 \text { pacientes submetidos a bolsa } \\
\text { de colostomia após cirurgia de } \\
\text { câncer colorretal }\end{array}$ & $\begin{array}{l}\text { Estudo de } \\
\text { Caso }\end{array}$ & Brasil \\
\hline
\end{tabular}

Fonte: Bitencourt EG, et al., 2020 
No tocante ao delineamento dos estudos, as abordagens de pesquisa eram descritivas qualitativas em sua maioria $(27,7 \%)$. Seguida das descritivo-exploratórias qualitativas e estudos de caso (16,6\%), bem como observacionais $(11,1 \%)$. Os demais tinham abordagem descritivo-exploratória quantitativa, transversaldescritiva quantitativa, descritiva exploratória, exploratória qualitativa, e descritiva correlacional, correspondendo a 5,6\% conforme explícito no Gráfico 1.

Gráfico 1 - Delineamento dos artigos quanto à abordagem de pesquisa

\section{Delineamento dos artigos}

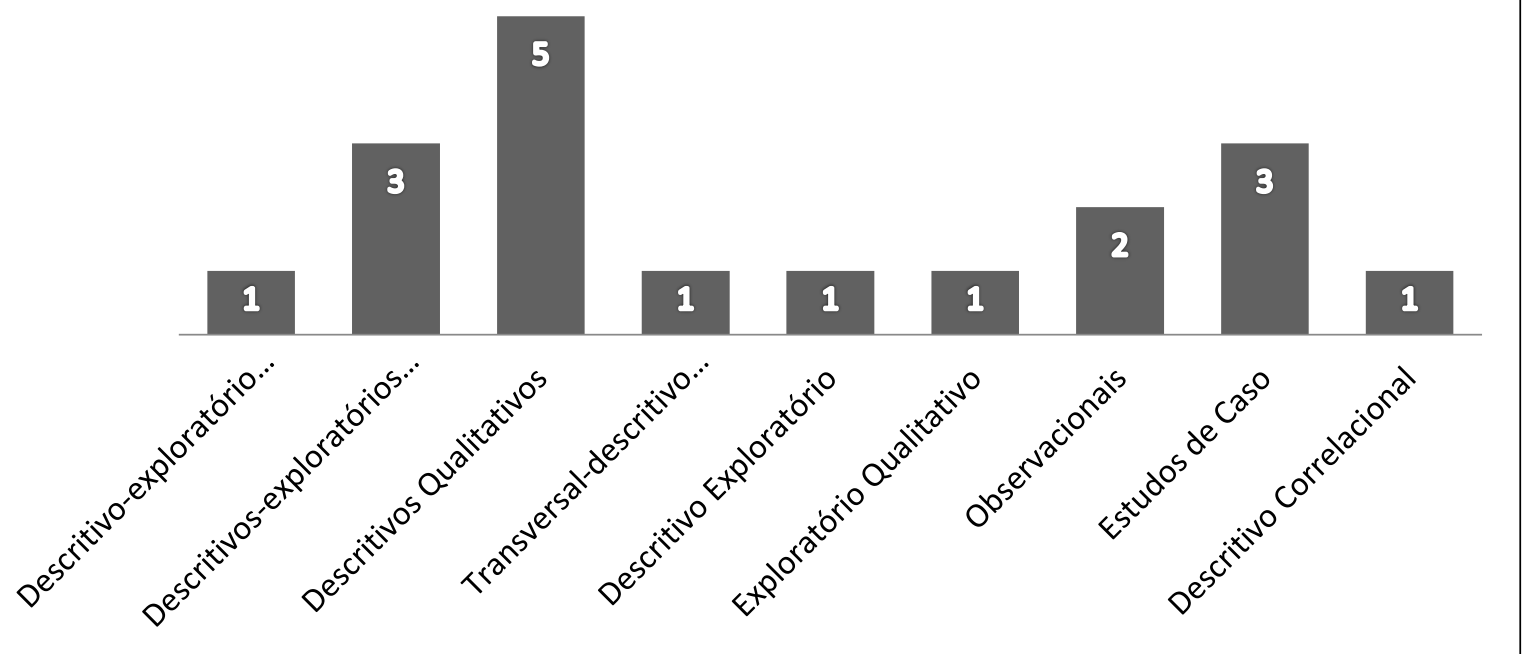

Fonte: Bitencourt EG, et al., 2020.

Com relação às amostras, $77,8 \%$ (14) dos estudos analisam pacientes colostomizado devido à neoplasia colorretal, as Doenças Inflamatórias Intestinais (DII) como a doença de Crohn, colite ulcerosa; doenças hereditárias como a polipose familiar, entre outros. Os demais estudos analisam a enfermagem, a SAE, entre outros. Dos 14 estudos analisados $57,1 \%$ (8) especificam o gênero; o fator causal mais frequente para a confecção da estomia; a faixa etária dos indivíduos; e a classificação das estomias, dado que pode ser observado na Tabela 2.

Tabela 2 - Agrupamento de achados quanto ao gênero, fator causal mais frequente, incidência por gênero, faixa etária estudada e classificação da colostomia com base nos artigos.

\begin{tabular}{cccccccccccc}
\hline \multirow{2}{*}{$\mathbf{N}^{*}$} & $\begin{array}{c}\text { Indivíduos } \\
\text { Estudados }\end{array}$ & \multicolumn{2}{c}{ Gêneros } & \multicolumn{2}{c}{$\begin{array}{c}\text { Câncer } \\
\text { colorretal }\end{array}$} & \multicolumn{2}{c}{$\begin{array}{c}\text { Porcentagem } \\
\text { de Incidencia }\end{array}$} & \multicolumn{2}{c}{$\begin{array}{c}\text { Faixa } \\
\text { etária }\end{array}$} & \multicolumn{2}{c}{ Classificação } \\
\cline { 2 - 12 } & $\mathbf{M}^{\star *}$ & $\mathbf{F}^{\star * *}$ & $\mathbf{M}$ & $\mathbf{F}$ & $\mathbf{M}$ & $\mathbf{F}$ & $\mathbf{M}$ & $\mathbf{F}$ & Temporária & Definitiva \\
\hline 2 & 21 & 12 & 9 & 9 & 5 & $43 \%$ & $24 \%$ & $25-75$ & 7 & 7 \\
\hline 3 & 10 & 4 & 6 & 2 & 4 & $20 \%$ & $40 \%$ & $37-65$ & 4 & 6 \\
\hline 6 & 10 & 8 & 2 & 8 & 2 & $80 \%$ & $20 \%$ & $24-84$ & 0 & 10 \\
\hline 7 & 1 & 0 & 1 & 0 & 1 & $0 \%$ & $100 \%$ & 21 & 1 & 0 \\
\hline 9 & 21 & 10 & 11 & 10 & 11 & $48 \%$ & $52 \%$ & $29-54$ & 0 & 21 \\
\hline 11 & 13 & 3 & 10 & 3 & 10 & $23 \%$ & $77 \%$ & $18-79$ & 0 & 13 \\
\hline 13 & 45 & 27 & 18 & 14 & 11 & $31 \%$ & $24 \%$ & $30-65$ & 18 & 27 \\
\hline 14 & 123 & 63 & 60 & 25 & 24 & $20 \%$ & $20 \%$ & $61-92$ & 68 & 55 \\
\hline $\begin{array}{c}\text { Total } \\
\text { Geral }\end{array}$ & 244 & $52 \%$ & $48 \%$ & 71 & 68 & $29 \%$ & $28 \%$ & $18-92$ & 98 & 139 \\
\hline
\end{tabular}

Legenda: ${ }^{*}$ Corresponde ao número do estudo na figura $1 ;{ }^{* *}$ Corresponde ao Gênero Masculino; ${ }^{* \star *}$ Corresponde ao Gênero Feminino. Fonte: Bitencourt EG, et al., 2020.

Com base nos artigos analisados e segundo o IBGE, a população do Brasil chegou a 211,8 milhões de habitantes em julho de 2020 (IBGE, 2020). Estimam-se, para cada ano do triênio de 2020-2022, sejam 
diagnosticados 20.540 casos de câncer de cólon e reto em homens e 20.470 em mulheres. Esses valores correspondem a um risco estimado de 19,64 casos novos a cada 100 mil homens e 19,03 para cada 100 mil mulheres (INCA, 2020).

$\mathrm{Na}$ construção do estudo, buscou-se identificar qual tipo de pesquisa embasaria melhor o artigo, assim optamos por pesquisas qualitativas que nos mostraram um retrato mais pessoal a respeito da vida dos indivíduos, como entrevistas, relatos pessoais e os estudos de casos clínicos (SONOBE HM, et al., 2002). Eles nos permitiram a compreensão do conceito da sistematização da assistência e a visão holística a respeito do paciente (CACAIS AFMV, et al., 2007; FREITAS MRI, et al., 2000). Um exemplo foi um estudo clínico a respeito de uma jovem que aos 21 anos foi atendida em três consultas, sendo a primeira para coleta de dados, a segunda consulta com o objetivo de realizar intervenção, e a última a avaliação dos resultados e a educação permanente, baseado na teoria de Orem - Autocuidado (SAMPAIO FAA, et al., 2008).

Os estudos encontrados foram agrupados empiricamente com base em seu conteúdo: autoaceitação do sujeito e/ou parceiro, reintegração ao meio laboral, familiar e ressocialização, autoimagem, resiliência, assistência, sistematização, acolhimento, educação permanente, visão holística e correlacionados aos fatores biológicos, psicológicos e sociais.

A sexualidade na juventude vem como uma forma de liberdade e afirmação de independência, geralmente, o jovem se preocupa em agradar, ser atraente, despertar desejo e ter uma boa performance, porém é indispensável que haja estabilidade psicóloga e autoaceitação para que a prática seja saudável (FREITAS MRI, et al., 2000; GOULART MB, et al., 2017).

Pacientes que não suportam penetração vaginal podem buscar prazer em outras partes do corpo (como seios, coxas ou até mesmo por meio da masturbação). Frequentemente, o sexo é substituído por gestos de afeto, companheirismo e amor. Estas são adaptações que substituem as relações sexuais regulares (FREITAS MRI e PELÁ NTR, 2000).

A repetição de experiências negativas durante a relação sexual aumentará a frustração, a decepção e a exigência, o que ainda fará com que as pessoas temam a próxima relação sexual, principalmente devido ao fato de os homens associarem a sexualidade ao comportamento sexual por meio da penetração. A preocupação com o desempenho, o medo do fracasso e o medo de novas frustrações prejudicam a autoestima e a autoconfiança dos homens e, por vezes, acabam prejudicando sua saúde emocional (GALATI MCR, 2014).

Os desafios na vida adulta não estão muito distantes dos vividos pelos jovens, entretanto no que diz respeito às intimidades sexuais, os homens relatam impotência sexual, perda de libido e perda da ejaculação, já as mulheres, desconforto e ressecamento vaginal, sendo a principal causa os fatores psicólogos, atrelados ao medo, ansiedade, sensação de sujeira e repugnância durante 0 ato sexual. A partir desses desafios há necessidade de se reinventar em todas as esferas da vida, tentar viver com maior qualidade e ressignificar o processo de vida para se adaptar e conseguir sanar as demandas sociais de um ser produtivo, como trabalhar (uma vez que não se sentem aptos para tal) e ter relações sociais e afetivas (SANTOS FS, et al., 2019).

Quanto a integração social, procurou-se compreender como os indivíduos têm conseguido ressignificar os processos de vida diante de um desafio tão grande que é a convivência e adaptação à colostomia e as implicações nas esferas biológicas, psicológicas e sociais, principalmente na fase de transição entre a juventude e a vida adulta, que naturalmente exige uma mudança na mentalidade (DE PAULA MAB, et al. 2009; MIRANDA LSG, et al., 2018).

Muitas vezes os estomizados deixam de realizar as refeições em locais públicos, devido ao medo de passar vergonha por causa das eliminações de gases, odor característico, receio da desconexão do dispositivo com extravasamento de fezes em locais de grande circulação de pessoas, relacionado à má qualidade dos dispositivos, contribuindo para a atitude de isolamento social, com intuito de atenuar os sentimentos de rejeição, constrangimento, insegurança e medo (SAMPAIO FAA, et al., 2008; SILVA AL, et al., 2006). 
Ter uma rede de apoio bem estruturada e educada é de suma importância para auxiliar o paciente em seu processo de autoaceitação e recuperação que culminará no autocuidado com qualidade, visto que os colostomizados dependem emocionalmente e fisicamente do suporte de seus familiares ou cuidadores (SANTOS FS, et al., 2019; BARBOSA MH, et al., 2014).

A juventude é uma fase de transição que acaba acarretando em diversas transformações no corpo e na formação da personalidade. Além de lidar com essas mudanças, o jovem colostomizado ainda precisa lidar com essa nova realidade, tendo que enfrentar os desafios da transição da juventude para a vida adulta, desenvolvendo sua sexualidade, ambiência social e superação de preconceitos (SELAU CM, et al., 2019). Como possibilidade para o fortalecimento da autoestima, trabalhar a autoimagem é essencial para o aumento da qualidade de vida, uma vez que a bolsa se trata de um dispositivo anexo ao corpo que acompanhará o indivíduo enquanto esse permanecer estomizado (BATISTA MRFF, et al., 2011). Não deixar se abater e ser resiliente é indispensável, visto que os sentimentos envolvidos sobre a bolsa coletora se mostram em sua maioria de cunho negativo (COELHO AR, et al., 2013).

O enfermeiro, como membro ativo do corpo multidisciplinar, tem papel importante no processo de educação do estomizado, pois lança mão de competência técnica e científica, além de ferramentas que viabilizam a assistência, por exemplo, a sistematização da assistência de enfermagem, que através dos diagnósticos e prescrição de enfermagem possibilitará estabelecer e elencar as dificuldades relacionadas a tríade: readaptação, ressocialização e reabilitação. Portanto, o enfermeiro acompanha, evolui e evidencia o prognóstico de seu paciente, reavaliando de forma contínua o surgimento de dificuldades que possam aparecer (MAURICIO VC, et al., 2013; BATISTA MRFF, et al., 2011).

A equipe de enfermagem necessita de educação permanente no que diz respeito aos cuidados com a colostomia, para estabelecer um plano de cuidados baseados em evidências científicas e orientar o paciente, com uma abordagem humanizada, permitindo que o mesmo se sinta acolhido e encorajado a esclarecer suas dúvidas e expressar seus sentimentos (VIEIRA LM, et al., 2013; GOULART MB, et al., 2017).

Ao decorrer do processo de adaptação, é indispensável o suporte e atuação da equipe transdisciplinar (OLIVEIRA IV, et al., 2018). No que se refere ao corpo de enfermagem, a mesma precisa colaborar para a ressocialização do estomizado, bem como para o restabelecimento de suas necessidades físicas e psicológicas, dentre outras (BARBUTTI RCS, et al., 2008).

Ao assistir ao paciente estomizado, é crucial que se estabeleçam boas estratégias de cunho educacional, com características de permanência e esclarecimentos, para sanar suas necessidades particulares, assim como de seus familiares e ajudadores, com foco tanto na reabilitação, quando a promoção da elevação na qualidade de vida e aporte para sofrimentos de caráter emocional que ele possa enfrentar em sua adaptação (MAURICIO VC, et al., 2013; BARBUTTI RCS, et al., 2008).

O profissional enfermeiro deve gerenciar de forma holística o paciente estomizado, dando importância às demandas físicas, psicológicas, espirituais e sociais do indivíduo (MIRANDA LSG, et al., 2018). O papel de educador é também muito importante, uma vez que é imprescindível reabilitar e ressocializar o paciente colostomizado (SILVA ES, et al., 2015).

Infelizmente o conteúdo que gera embasamento científico para realizar um tratamento que supra as demandas do público-alvo deste artigo, os jovens adultos (18-29 anos), é escasso, o que inviabiliza um maior aprofundamento teórico que inevitavelmente refletirá na assistência prestada (OLIVEIRA IV, et al., 2018). A partir daí é imprescindível que os serviços de saúde juntamente com a equipe transdisciplinar sejam capazes de planejar uma assistência embasada em apoio psicológico, educacional e reabilitação, para que a qualidade de vida melhore significativamente (SILVA ES, et al., 2015).

\section{CONSIDERAÇÕES FINAIS}

O indivíduo que é submetido à confecção de uma colostomia, terá que se readaptar as diversas alterações que se manifestam, desde mudanças na fisiologia intestinal, na autoestima e readaptação corporal. Isso interfere de forma direta em suas relações familiares, afetivas, sociais e de trabalho. Outro fator de extrema 
relevância é o aspecto psicológico e o desafio de se autoaceitar e conviver diante dos inúmeros questionamentos práticos relacionados à vida cotidiana, como a viabilidade de realizar o auto cuidado $\mathrm{e}$ manutenção de suas atividades sociais e laborais, visto que a resposta aos cuidados ofertados estará intimamente ligada a adaptação ao estoma, associado à disposição e aptidão do indivíduo e de seus cuidadores, uma vez que o uso da bolsa coletora reverbera sentimentos majoritariamente negativos de desesperança, medo, rejeição, depressão, mutilação e dor. Ao enfermeiro cabe atentar o indivíduo como um todo, respeitando o conceito biopsicossocial e tendo uma visão holística, para finalmente atender integralmente as necessidades do ser humano.

\section{REFERÊNCIAS}

1. BACELAR S, et al. Expressões médicas: falhas e acertos. Acta Cirúrgica Brasileira, 2004; 19(5): 582-4.

2. BARBOSA MH, et al. Aspectos clínicos e epidemiológicos de estomizados intestinais de um município de Minas gerais. Revista de Enfermagem e Atenção à Saúde (REAS), 2014; 3(1): 64-73.

3. BARBUTTI RCS, et al. Ostomia, uma difícil adaptação. Rev. SBPH, 2008; 11(2): 27-39.

4. BATISTA MRFF, et al. Autoimagem de clientes com colostomia em relação à bolsa coletora. Rev. Bras Enferm. (REBEn), 2011; 64(6): 1043-7.

5. BONILL-DE-LAS-NIEVES C, et al. Convivendo com estomas digestivos: estratégias de enfrentamento da nova realidade física. Rev. Latino-Am Enfermagem (RLAE), 2014; 22(3): 394-400.

6. CASCAIS AFMV, et al. O impacto da ostomia no processo de viver humano. Texto Contexto Enferm., 2007; 16(1): 163-7.

7. COELHO AR, et al. A estomia mudando a vida: enfrentar para viver. Rev. Min. Enferm. (REME), 2013; 17(2): 258267.

8. PAULA MAB, et al. Os Significados da Sexualidade para a Pessoa com Estoma Intestinal Definitivo. Rev. Bras Coloproct, 2009; 29(1): 077-082.

9. ESTIMA, R. Entrevista. Estima - Brazilian Journal of Enterostomal Therapy, 2004; 2(1).

10. FREIRE DA, et al. Autoimagem e autocuidado na vivencia de pacientes estomizados: o olhar da Enfermagem. Rev Min Enferm. (REME), 2017; 21: e-1029.

11. FREITAS MRI, PELÁ NTR. Subsídios para compreensão da sexualidade do parceiro do sujeito portador de colostomia definitiva. Rev. Latino-Am Enfermagem, 2000; 8(5): 28-33.

12. GALATI MCR, et al. Sexualidade e qualidade de vida em homens com dificuldades sexuais. Psico-USF. 2014; 19(2): 242-52.

13. GOULART MB, et al. A sexualidade do paciente estomizado no discurso do enfermeiro. Rev. Min Enferm., 2017; 21: e-1041.

14. IBGE - Instituto Brasileiro de Geografia e Estatística. IBGE divulga estimativa da população dos municípios para 2020. Diário Oficial da União (D.O.U.), 2020.

15. MINISTÉRIO DA SAÚDE - MS. Instituto Nacional de Câncer. Estimativas 2020 - Síntese de resultados e comentários. Instituto Nacional de Câncer. INCA, Brasil. 2020.

16. MAURÍCIO VC, et al. O enfermeiro e sua participação no processo de reabilitação da pessoa com estoma. Esc Anna Nery, 2013; 17(3): 416-22.

17. DURAN ECM, TOLEDO VP. Análise da produção do conhecimento em processo de enfermagem: estudo exploratório-descritivo. Rev Gaúcha Enferm., 2011; 32(2): 234-40.

18. MEIREILES CA, et al. Estudo teórico da demarcação do estoma intestinal. R. Bras Enferm., 2001; 54(3): 500-10.

19. MIRANDA LSG, et al. Qualidade de vida da pessoa estomizada: relação com os cuidados prestados na consulta de enfermagem com a estomaterapia. Escola Anna Nery, 2018; 22(4).

20. NASCIMENTO CMS, et al. Vivência do paciente estomizado: uma contribuição para a assistência de enfermagem. Texto Contexto Enferm., 2011; 20(3): 557-64.

21. OLIVEIRA IV, et al. Cuidado e saúde em pacientes estomizados. Rev. Bras Promoç Saúde, 2018; 31(2): 1-9.

22. RAITZ TR, PETTERS LCF. Novos desafios dos jovens na atualidade: trabalho, educação e família. Psicologia \& Sociedade, 2008; 20(3): 408-16.

23. SAMPAIO FAA, et al. Assistência de enfermagem a paciente com colostomia: aplicação da teoria de Orem*. ACTA Paul Enferm., 2008; 21(1): 94-100.

24. SANTOS FS, et al. Percepção dos cônjuges de pessoas com estomia intestinal sobre a sexualidade do casal. Rev. Min Enferm., 2019; 23: e-1217.

25. SELAU CM, et al. Percepção dos pacientes com estomia intestinal em relação às mudanças nutricionais e estilo de vida. Texto Contexto Enferm., 2019; 28: e20180156

26. SILVA AL, SHIMIZU HE. O significado da mudança no modo de vida da pessoa com estomia intestinal definitiva. Rev. Latino-Am Enfermagem, 2006; 14(4): 483-90.

27. SILVA ES, et al. Protocolo de enfermagem para as alterações psicossociais e espirituais da pessoa com colostomia. Cogitare Enferm., 2015; 20(3): 467-474.

28. SONOBE HM, et al. A visão do colostomizado sobre o uso da bolsa de colostomia. Revista Brasileira de Cancerologia, 2002; 48(3): 341-348.

29. SOUZA MT, et al. Revisão integrativa: o que é e como fazer. Einstein (São Paulo), 2010; 8(1): 102-6.

30. VIEIRA LM, et al. A. Câncer Colorretal: entre o sofrimento e o repensar na vida. Saúde em Debate, 2013: 37(97): 261-69. 